\title{
El papel de los bosques en el Protocolo de Kyoto: el caso del manejo forestal de Nueva Zelanda y México
}

\author{
Ana Bertha Cuevas Tello*
}

$\mathrm{D}$ e algunos años a la fecha el Protocolo de Kyoto ha sido objeto de análisis tanto de la esfera política como de los sectores económicos y académicos de la sociedad nacional e internacional, así como de los organismos no gubernamentales de ambas esferas. Su entrada en vigor, a principios de 2005, ha sido alabada por algunos y criticada por otros. Para los primeros el Protocolo representa el primer paso para revertir el proceso del cambio climático; para los segundos la intención es buena, sin embargo, los mecanismos para lograr el objetivo del acuerdo no son convincentes.

Existen muchos puntos de controversia en esta polémica; no obstante, el único que se analizará en este trabajo es el que concierne a los recursos forestales, elemento fundamental en el tratado por su dualidad en relación con el cambio climático, ya que a la vez que éste funge como sumidero de carbono y generador de oxígeno, al momento de su deforestación emite grandes cantidades de gases de efecto invernadero.

En este sentido, el objetivo de este ensayo es analizar en dónde radica la complejidad de los recursos forestales, por qué representan un papel importante dentro

* Profesora investigadora del Departamento de Estudios del Pacífico de la Universidad de Guadalajara. del objetivo de reducir las emisiones de gases, y por qué causa polémica su funcionalidad dentro del tratado. Posteriormente se aplica el análisis al estudio del manejo forestal de dos países: Nueva Zelanda y México, naciones seleccionadas por tener diferente uso de sus recursos forestales.

En el primer apartado se exponen las funciones de los bosques y la complejidad de su manejo. En el segundo se analizan, de manera general, el reconocimiento nacional e internacional de la importancia de los recursos forestales en el cambio climático y su papel fundamental en el Protocolo de Kyoto. En el tercero se examina el manejo real de los recursos forestales en Nueva Zelanda y México, para comprender lo complejo que puede ser, en la práctica, el mantener las funciones de los recursos forestales como un medio para detener el incremento de los gases de efecto invernadero. Por último, con el análisis teórico y práctico se establecerán las conclusiones.

\section{La función de los bosques y la complejidad de su manejo}

Los bosques absorben el dióxido de carbono y lo intercambian por oxigeno (purificación del aire vía fotosíntesis), regulan el balance del agua y la temperatura de la tierra (al permitir la infiltración de los mantos freáticos), generan la proliferación de una gran 
diversidad biológica, además de servir de recreación y de vista panorámica para la sociedad. ${ }^{1}$

Estas funciones vitales, que resulta esencial garantizarlas, cada día se hace más difícil conservarlas debido a que la cubierta forestal del planeta disminuye día con día; esto se debe a que la diversidad de los beneficios de los bosques y las selvas es inherente a múltiples intereses que impiden, de alguna manera, su natural permanencia; veamos una explicación teórica del porqué. Cuando los economistas analizan los recursos naturales establecen cuatro distinciones básicas: a) apropiables o inapropiables; b) renovables o no; c) esenciales o no esenciales; d) bienes públicos o privados. ${ }^{2}$

En este sentido, los recursos forestales pertenecen a todas las clasificaciones, excepto a la de bienes no esenciales. Respecto al punto b), los bosques son apropiables porque se recoge un valor económico de ellos, sus dueños pueden vender los árboles o la tierra bajo la ley de la oferta y la demanda, y obtener un beneficio monetario de esto. Al mismo tiempo son inapropiables porque su externalidades, negativas o positivas, van más allá del costo de la madera o del terreno. Es decir, nadie se puede apropiar del oxígeno limpio ni impedir que otros se beneficien de éste (no excluible), y nada puede someter el dióxido de carbono (que los bosques absorben en fotosíntesis y que emiten al ser deforestados) en un lugar determinado.

b) Los bosques y las selvas son renovables porque son un conjunto dinámico de diversas especies y sistemas sustentables de vida, los cuales tienen la capacidad de renovación propia, ${ }^{3}$ a la vez que se puede contribuir a su regeneración por medio de plantaciones forestales. $\mathrm{Al}$ mismo tiempo, no son renovables cuando la tala de árboles es superior a la capacidad de autorenova- ción de los bosques.

c) Son esenciales porque la vida depende invariablemente del oxígeno limpio, de la posición que ocupan en el ciclo del agua y porque cumplen un papel único en el hábitat de un sinfín de especies de flora y fauna. En este sentido, no existe nada ni nadie que pueda sustituir la función de éstos, es decir, la singularidad del los recursos forestales no tiene reemplazo.

d) Son bienes privados porque tienen dueños (gobierno, ejidos, firmas o particulares) que disponen de ellos y para quienes representan una inversión económica. Al mismo tiempo, son bienes públicos porque las externalidades (positivas o negativas) que generan van más allá de los propietarios, se difunden en forma indivisible por todo el planeta independientemente de que se esté consciente o no de ello. Así, al no poder limitar las externalidades a un solo país se afirma que los recursos forestales son bienes públicos mundiales, como la selva amazónica, considerada el pulmón del planeta.

Hasta aquí quedan claras dos situaciones: a) de las funciones básicas de los bosques y las selvas se preserva la vida, por lo que se debe procurar su permanencia, y b) los recursos forestales tienen un valor económico, lo que implica la existencia de un mercado competitivo que busca la optimación de los recursos y la ganancia monetaria. Estas dos caras de la misma moneda se materializan en dos niveles; por un lado, en el sistema internacional debido a que existen organismos y regímenes que por medio de investigaciones, análisis y acuerdos procuran el bienestar de los recursos forestales, mientras que a nivel doméstico se refuerza el propósito anterior con leyes forestales que tienen como principio el manejo sustentable de los mismos. Por otro lado, dentro del comercio 
mundial de bienes y servicios los productos forestales representan el 3 por ciento del total, esto sin contar las sumas generadas en la tala clandestina y el cambio de uso de la tierra forestal ${ }^{4}$ para otros fines, tales como la agricultura o la urbanización.

Estas dos realidades han sido tema de discusión entre los expertos con dos posturas diferentes: los defensores del medio ambiente y los protectores del comercio. Las razones que defiende cada uno de los grupos no se discutirá aquí, ya que no es el objetivo del trabajo; simplemente se señalan para exponer la complejidad del manejo de los recursos forestales y para entender el porqué si los recursos forestales son bienes esenciales, el área forestal se reduce día con día.

\section{El manejo sustentable de los recursos forestales y el Protocolo de Kyoto}

En 1990 los recursos forestales cubrían una superficie de 3,962 millones de hectáreas en todo el planeta; para el año 2000 esta cifra se redujo a 3,869 millones, es decir, en una década la cubierta de bosques y selvas disminuyó en poco más de 90 millones de hectáreas, lo que significa una reducción promedio anual de 0.2 por ciento, cifra alarmante si se considera el crecimiento de la población mundial y el incremento de dióxido de carbono en el planeta. Sin embargo, el problema de la reducción de la cubierta forestal no es un asunto de la última década del siglo xx, ya que desde 1947 la Organización de las Naciones Unidas para la Agricultura y la Alimentación (FAO, por su siglas en inglés) expresaba esta preocupación: "se continúa reduciendo la superficie forestal del mundo por la explotación inmoderada llevada a cabo sin pensar en el porvenir o en la regeneración de las reservas". ${ }^{5}$
Es decir, el interés por resguardar los recursos forestales no es asunto nuevo; de hecho, la comunidad científica desde los sesenta ha venido reportando los perjuicios ambientales (entre ellos los forestales), que si bien sirvieron para concientizar a cierto sector de la humanidad, para los gobiernos este tema no formaba parte de su agenda. Fue hasta finales de los ochenta cuando el fin de la Guerra Fría abrió paso a nuevos asuntos que en el pasado habían quedado relegados, entre ellos el del medio ambiente.

Con la conclusión del bipolarismo, los gobiernos nacionales en particular y la comunidad internacional en general empezaron a entender que al nuevo enemigo a vencer ya no se le combatía con armamento militar o con estrategias de guerra, ya que como Giddens lo señaló: "las naciones afrontan hoy riesgos y peligros en lugar de enemigos", ${ }^{6}$ entre ellos el deterioro ambiental en el que se encuentra el planeta.

Entre los primeros asuntos a los que el sistema internacional prestó atención, fue al cambio climático de la Tierra. En 1992, en la Conferencia de Río, celebrada en Brasil, se establecieron resultados concretos. Los dos convenios más importantes se refieren al cambio climático y a la declaración de los bosques. Como consecuencia de los productos anteriores, se desarrollaron dos sucesos, uno en sistema internacional y otro en los gobiernos locales:

1. De la Convención Marco de Naciones Unidas sobre el Cambio Climático (UNFCCC, por sus siglas en inglés) surgió el objetivo de "estabilizar las concentraciones de gases de efecto invernadero en la atmósfera, a un nivel que previniera una interferencia antropogénica peligrosa para el sistema climático. Este nivel debería alcanzarse a tiempo para que los ecosis- 
temas pudieran adaptarse naturalmente al cambio climático, de manera que la producción de alimentos no se viera amenazada y que fuera posible el desarrollo económico sustentable". ${ }^{7}$ Por lo tanto, los países desarrollados (por ser los emisores de más de 60 por ciento de los gases de efecto invernadero) se comprometieron a reducir las emisiones, en el año 2000, a los niveles que se tenían en los años noventa. Cinco años después, a falta de acuerdos y logros concretos, y con el fin de fortalecer el compromiso, en 1997 la Convención creó el Protocolo de Kyoto, cuyo objetivo fundamental fue comprometer a los países del llamado anexo I (en su mayoría desarrollados) a reducir, en el periodo 2008-2012, del total de las emisiones a la atmósfera por lo menos en 5.2 por ciento en relación con los niveles de $1990 .{ }^{8}$ Con la intención de alcanzar ese objetivo, debido a la complejidad que esto implicaba se crearon los mecanismos flexibles, los cuales permitirán alcanzar las metas acordadas sobre la base de proyectos de reducción de emisiones de efecto invernadero en terceros países (industrializados o en vías de desarrollo), comercializando los certificados de emisiones o reduciendo el porcentaje de gases de carbono en el aire mediante el desarrollo de bosques o zonas de vegetación. ${ }^{9}$

Aquí se llega a un punto importante que exige reflexionar sobre el cambio climático, los recursos forestales y su relación con el Protocolo de Kyoto. Primero, los gases de efecto invernadero, en cantidades moderadas, desempeñan un papel importante en el clima de la Tierra debido a que los que se producen naturalmente impiden que parte del calor solar regrese al espacio (sin ellos el planeta sería un lugar demasiado frío para generar vida); empero, cuando el volumen de éstos gases es considerable y crece sin control, provo- can temperaturas artificialmente elevadas y modifican drásticamente el clima, con consecuencias nefastas tales como: lluvias intensas, inundaciones y sequías, temperaturas climáticas extremas, aumento del nivel del mar, extinción de especies de flora y fauna, disminución de los rendimientos agrícolas (especialmente en las zonas tropicales y subtropicales), perturbaciones en el aprovechamiento de la tierra y el suministro de alimentos, entre otros. ${ }^{10}$

Segundo, desde principios de siglo xx la concentración de gases de efecto invernadero, especialmente de dióxido de carbono, está aumentando como consecuencia del desarrollo de la actividad humana, la industrialización y los avances tecnológicos. La quema de combustibles fósiles y biomasa (gas natural, petróleo, combustibles, leña) en procesos industriales, transporte y actividades domésticas (cocina, calefacción, entre otros), junto con los incendios forestales (y de pastizales), más la deforestación de los bosques y selvas contribuyen al aumento descontrolado de los gases de efecto invernadero en el mundo. ${ }^{11}$

Tercero, los dos puntos anteriores fueron objeto de reflexión desde la UNFCCC y maduraron en el Protocolo de Kyoto, ya que explícitamente se reconoció que el mejoramiento de los sumideros de carbono y la reducción de las emisiones presentaban un potencial para hacer frente al cambio climático. A partir de aquí los bosques se convirtieron en un elemento clave.

Para hacer frente al cambio climático las estrategias que se establecieron fueron: la mitigación y la adaptación. La primera implica "la intervención del hombre concebida para reducir la emisión de gases de efecto invernadero en sus fuentes de origen o para incrementar los sumideros de carbono". ${ }^{12}$ La segunda "designa los ajustes en los sistemas naturales o humanos en 
respuesta a estímulos climáticos o a sus efectos, con el fin de disminuir el daño que ocasionan o aprovechar los beneficios". ${ }^{13}$ Ambas medidas se refuerzan entre sí; no obstante, la primera subraya el papel de los generadores o mitigadores del problema, y la segunda el hacer frente $\mathrm{u}$ obtener provecho de los cambios ocasionados. Entre las medidas de mitigación se consideraron los mecanismos flexibles (participar en proyectos de inversión que impliquen, entre otros aspectos, la reforestación y la forestación) y las medidas internas (disminución de las actividades contaminantes o la utilización de energía renovable, más los marcos legales). Es decir, dentro de la estrategia de mitigación los recursos forestales son esenciales, ya que éstos fungen como sumideros, por lo que los 143 países signatarios buscarán su extensión.

2. La Declaración de los Bosques es un producto de la Conferencia de Río que establece principios para un consenso mundial respecto de la ordenación, la conservación y el desarrollo sostenible de los bosques. Ésta vino a consolidar el compromiso de prestar mayor atención a los bosques y a considerar este recurso como un bien público global y como un recurso natural al que se le debe brindar un manejo sustentable. No obstante lo anterior, esta Declaración no tiene fuerza jurídica obligatoria.

A partir de este encuentro los países miembros de Naciones Unidas se compro- metieron a emitir o a reformar las leyes forestales en sus respectivas naciones. El objetivo principal de estas leyes debía ser manejar en forma sustentable a los bosques, como la única vía de combinar el lado económico con el ambiental.

Desde un punto de vista teórico, si partimos del hecho de que el manejo sustentable significa "satisfacer las necesidades del presente, sin comprometer la capacidad de las generaciones futuras de satisfacer sus propias necesidades", ${ }^{14}$ entonces lo que las autoridades correspondientes deben buscar es una combinación entre la producción forestal y la permanencia de los bosques, es decir, lo que el concepto propone es la existencia del mercado sin perjudicar el medio ambiente. La curva de posibilidades de la producción entre la generación actual y la futura lo ilustrará de mejor manera.

El panel (a) muestra la relación de intercambio a la que se enfrenta nuestra generación en la actualidad. El panel (b) muestra las curvas de posibilidad de producción de las futuras generaciones. Según el panel (a), la generación presente podría elegir las combinaciones (c , e ), (c , e ) o cualquier otra curva. Lo que $\mathrm{se}^{1} \mathrm{deb}^{2}$ rêsaltar es que el manejo forestal del presente está conectado con lo que sucederá en el futuro. Es razonable suponer que la degradación ambiental de hoy afectará las posibilidades futuras, lo que generaría que 


\section{Gráfica 1}

Curvas de posibilidades de la producción (CPP) para la generación actual y la futura

(a)

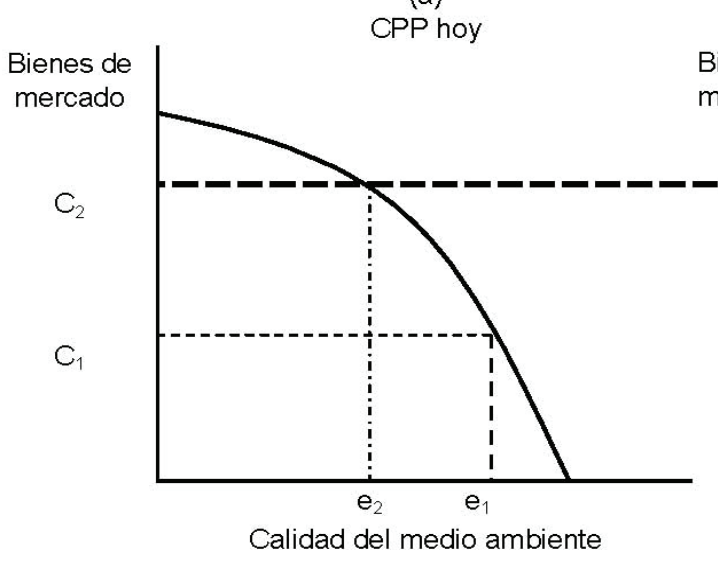

(b)

CPP dentro de 60 años

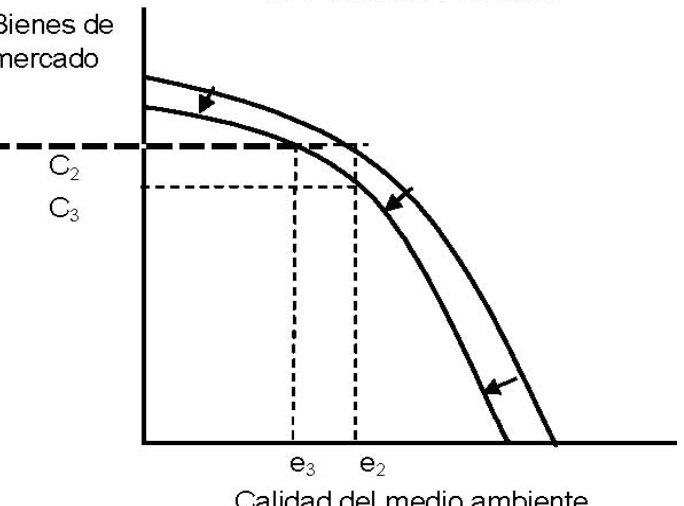

Fuente: Barry C. Field y Martha K. Field, 2003, Economía ambiental, McGraw-Hill, México.

la CPP se desplazara hacia la izquierda y hacia abajo. En este sentido, las generaciones del futuro podrían gozar del mismo nivel de producción del mercado que tenemos en el presente (c ) pero con un medio ambiente de menor călidad que el actual; o bien podrían beneficiarse de calidad ambiental similar pero con un nivel menor de producción (c).

Se debe aclarar que efectuar un análisis del futuro es mucho más complejo que lo analizado, debido a que "las condiciones futuras no dependen únicamente de la degradación ambiental, sino también de los avances técnicos y de cómo varíen las habilidades humanas". ${ }^{15}$ Sin embargo, el hecho que se busca destacar en la idea de desarrollo sustentable es que las acciones y las decisiones que se realizan hoy tienen repercusiones en el futuro.

Es decir, el objetivo del principio del desarrollo sustentable es evitar que las curvas futuras de posibilidades de producción se desplacen hacia abajo y a la izquierda. Lo anterior no significa que se tenga que maximizar la calidad ambiental hoy, porque equivaldría a una producción nula de bienes y servicios (en este caso de la industria forestal); lo que significa es que los efectos actuales sobre el medio ambiente tienen que ser los suficientemente moderados como para evitar reducir el bienestar del medio ambiente de las generaciones futuras. ${ }^{16}$

En el caso de los recursos forestales, como en el de cualquier elemento natural, mientras más pronto se tomen acciones mejores resultados de bienestar y producción tendrán las generaciones futuras. Para el caso concreto de los recursos forestales la FAO propone, por un lado, "evitar la deforestación, mejorar la eficiencia de utilización de combustible, mejorar los métodos de tala o apeo de árboles", y por otro, "mejorar el manejo de los bosques, establecer reservas y áreas protegidas, replantear los usos de los bosques, reducir la necesidades de deforestación y, por último, abocarse a la forestación, la reforestación, la agrosilvicultura y el arbolado urbano". ${ }^{17}$

En el desarrollo del apartado queda claro porqué los recursos forestales son 
esenciales en el marco del Protocolo de Kyoto y el porqué en la primera mitad de la década de los noventa se reformaron y se crearon legislaturas forestales con fines de desarrollo sustentable. Sin embargo, a más de una década las tasas de deforestación continúan, por lo que surge la pregunta: ¿están funcionando las medidas de desarrollo forestal sustentable? ¿Bajo qué variables podemos medir la funcionalidad del manejo sustentable en algunos países? Para tratar de responder lo anterior propongo efectuar una revisión práctica de dos países: Nueva Zelanda y México. El análisis comparativo de estas dos naciones se establecerá con base en variables cuantitativas como superficie forestal, tasa de deforestación, comercio forestal internacional, producción, tenencia de la cubierta forestal y plantaciones forestales.

\section{El manejo forestal en Nueva Zelanda y México}

El 30 por ciento de la superficie terrestre está cubierto de árboles, 56 por ciento está compuesto de bosques tropicales y subtropicales (selvas) y el resto por bosques templados y boreales. El 95 por ciento es bosque natural y el 5 por ciento son plantaciones. ${ }^{18}$ Los países con mayor superficie forestal son Rusia, Brasil, Canadá, Estados Unidos, China e Indonesia.

México y Nueva Zelanda son países que, en relación con su tamaño, cuentan con moderada superficie forestal, ya que para ambos representa alrededor de 30 por ciento de su superficie total. Sin embargo, en números absolutos México cuenta con mayor área forestal que Nueva Zelanda, ya que la cubierta forestal de este último representa 14 por ciento de la superficie forestal de México (de hecho, este última economía se encuentra entre los 30 países con mayor cobertura forestal en el mundo).

El cuadro 1 esboza el escenario de partida para el análisis de la situación forestal de estas dos economías. Se puede apreciar que en el periodo 1990-2000 Nueva Zelanda, bajo reformas efectuadas desde mediados de los ochenta y que se concretaron en 1993 con la enmienda a la ley forestal que enfatizaba el manejo sustentable de los bosques ${ }^{19}$ que llevaron a emprender planes y medidas concretas, tuvo un manejo positivo en la cubierta forestal. Es decir, en los noventa en este país no se presentaron tasas de deforestación; al contrario, aumentaron la cubierta forestal en 0.05 por ciento promedio anual. Caso diferente al de México, país que en el mismo periodo presentó una tasa de deforestación anual de 1.1 por ciento; es decir, mientras que el bosque de Nueva Zelanda crecía 39 mil hectáreas cada año, en México se disminuía 631 mil hectáreas de bosque anualmente. Este resultado es relativamente alto si se considera que este país desde 1992 reformó su Ley Forestal con base en el manejo sostenible de los

Cuadro 1

\begin{tabular}{lccccc}
\hline Países & $\begin{array}{c}\text { Superficie } \\
\text { (miles de } \\
\text { hect.) }\end{array}$ & $\begin{array}{c}\text { Superficie } \\
\text { forestal } \\
\text { (miles de hect.) }\end{array}$ & $\begin{array}{c}\text { Superficie } \\
\text { forestal }\end{array}$ & $\begin{array}{c}\text { Promedio anual } \\
\text { de deforestación }\end{array}$ & $\begin{array}{c}\text { Promedio anual } \\
\text { de deforestación } \\
1990-2000 \text { (miles de hect.) }\end{array}$ \\
$\begin{array}{lccccc}1990-2000(\%) \\
\text { Nueva Zelanda }\end{array}$ & 26,799 & 7,946 & 28.9 & -39 & -.05 \\
México & 190,869 & 55,205 & 29.7 & 631 & 1.08 \\
\hline
\end{tabular}

Fuente: www.fao.org/forestry 
bosques, es decir, contó con ocho años para que por medio de planes específicos disminuyera la deforestación; sin embargo, los resultados presentados indican la ausencia de esta práctica sustentable.

En suma, estos dos países, a partir de la Conferencia de 1992 como miembros activos de las Naciones Unidas, establecieron enmiendas en sus respectivos edictos legales. Sin embargo, durante la década de los noventa los dos mostraron resultados diferentes. Actualmente en Nueva Zelanda 5 por ciento del área forestal está constituida por plantaciones forestales, por que permiten el abastecimiento de 99 por ciento de la producción. Mientras que en México el suministro de la producción forestal se basa en su totalidad en los bosques naturales. ${ }^{20}$ Hasta 1996 no había regulaciones efectivas que establecieran un programa de plantaciones forestales en México, sino que fue hasta la reforma de 1997 cuando se implementó esta actividad, con la específica aclaración de que éstas serían llevadas a cabo en superficie no forestal (respetando los bosques naturales). Según cifras de la FAo, hasta el año 2004 se levantó la primera cosecha de las plantaciones, ${ }^{21}$ sin embargo, con relación a esto último, el Movimiento Mundial por los Bosques señala que hoy día más de 70 por ciento de la plantaciones se encuentran en mal estado y su nivel de producción es inferior a lo esperado. ${ }^{22}$

Entre los principales factores que provocan la disminución de la cubierta forestal se encuentran: la expansión agrícola (ésta se ha incrementando en casi el 70 por ciento de los países), ${ }^{23}$ el crecimiento de la zona urbana, los incendios, el pastoreo, la tala clandestina y el uso comercial. Aquí sólo se analizarán el primero y el último.

Como se puede apreciar en el cuadro 2 , la superficie agrícola de ambas nacio-
Cuadro 2

Cambio en la superficie agricola de Nueva Zelanda y México: 1990-2000 (miles de hectáreas)

\begin{tabular}{lcc}
\hline Año/crec. & Nueva Zelanda & México \\
\hline 1990 & 17,355 & 103,400 \\
1991 & 17,624 & 104,000 \\
1992 & 17,300 & 104,700 \\
1993 & 17,336 & 105,700 \\
1994 & 16,607 & 106,500 \\
1995 & 16,578 & 107,200 \\
1996 & 16,578 & 107,200 \\
1997 & 16,580 & 107,200 \\
1998 & 16,580 & 107,200 \\
1999 & 16,580 & 107,200 \\
2000 & 17,204 & 107,300 \\
Crec. Prom. Anval & -15 & 390 \\
cifras absolutas & & \\
Crec. Prom. Anual & -0.1 & 0.4 \\
(\%) & & \\
\hline
\end{tabular}

Fuente: www.fao.org/forestry

nes presentó modificaciones durante los noventa. Para Nueva Zelanda ésta se redujo en 15 mil hectáreas y para México aumentó en 390 mil hectáreas en promedio anualmente. Si comparamos los cambios en la superficie forestal y en la agrícola, se puede observar cierta relación; en Nueva Zelanda la primera aumentó y la segunda disminuyó, lo que se podría interpretar como que una proporción de esta última ha sido utilizada para plantaciones forestales. En México, mientras que el área de la tierra destinada a la agricultura aumentó, la cubierta forestal disminuyó; por lo tanto, según las cifras 62 por ciento de la disminución de la cubierta forestal fue utilizada para fines agrícolas y el resto en área de urbanización o incendios forestales, entre otras.

En cuanto al uso comercial de los recursos forestales, Nueva Zelanda tiene un mecanismo de producción efectivo, es decir, este país posee una perfecta coor- 
Cuadro 3

Producción de la industria forestal

\begin{tabular}{lcccc}
\hline Año & \multicolumn{2}{c}{ Nueva Zelanda } & \multicolumn{2}{c}{ México } \\
\cline { 2 - 5 } & $\begin{array}{c}\text { Metros } \\
\text { cúbicos }\end{array}$ & $\begin{array}{c}\text { Toneladas } \\
\text { métricas }\end{array}$ & $\begin{array}{c}\text { Metros } \\
\text { cúbicos }\end{array}$ & $\begin{array}{c}\text { Toneladas } \\
\text { métricas }\end{array}$ \\
\hline 1990 & $41,719,000$ & $14,507,000$ & $29,457,786$ & $24,378,000$ \\
1991 & $49,048,000$ & $15,807,000$ & $28,764,405$ & $23,952,000$ \\
1992 & $51,567,000$ & $14,500,000$ & $28,048,481$ & $22,591,000$ \\
1993 & $55,322,000$ & $15,884,000$ & $24,017,000$ & $19,138,000$ \\
1994 & $56,553,000$ & $16,043,000$ & $24,490,000$ & $19,134,000$ \\
1995 & $58,360,000$ & $16,552,000$ & $24,015,600$ & $23,865,200$ \\
1996 & $56,639,000$ & $15,916,000$ & $25,563,000$ & $24,594,000$ \\
1997 & $59,456,000$ & $16,463,000$ & $28,673,600$ & $26,603,400$ \\
1998 & $51,370,000$ & $16,392,000$ & $29,962,000$ & $31,691,000$ \\
1999 & $59,146,000$ & $16,897,000$ & $30,440,564$ & $31,798,993$ \\
2000 & $64,251,000$ & $18,677,000$ & $30,573,664$ & $32,597,000$ \\
\hline Crec. Prom. Anual (\%) & 4.4 & 2.6 & 0.4 & 2.9 \\
\hline
\end{tabular}

Fuente: www.fao.org/FAOSTA.

dinación en la cadena productiva de este recurso, mientras que en México se carece de la misma. En primer lugar, en Nueva Zelanda la industria maderera obtiene su insumo exclusivamente de plantaciones forestales y no de los bosques naturales, caso contrario al de México.

El cuadro 3 muestra, para cada país, la producción de la industria de bienes intermedios en general (primaria); la segunda proviene de la industria de la transformación de la madera (secundaria). Se aprecia en el cuadro que, aunque Nueva Zelanda es eficiente en ambas industrias (por mantener un crecimiento constante), la industria con menor valor agregado es la más fuerte en este país, pues tiene una producción tres veces mayor que la segunda; a diferencia de México, donde ambas industrias tiene una producción similar, aunque la producción de la industria secundaria registra mejor récord en cuanto al crecimiento.

Se observa que Nueva Zelanda (con menor superficie forestal disponible para uso comercial) tiene más del doble de la producción de México dentro de la industria forestal primaria. Mientras que en la industria forestal secundaria México tiene mayores volúmenes de producción que Nueva Zelanda, sin llegar a representar cantidades elevadas.

En cuanto al comercio internacional de la madera (cuadro 4), también encontramos datos interesantes. Hasta el año 2000 México mantuvo un comercio internacional de productos forestales de casi dos veces el de Nueva Zelanda; sin embargo, poco más de 90 por ciento de éste está integrado por importaciones; caso contrario al de Nueva Zelanda, en el cual 84 por ciento de su comercio son exportaciones, lo que explica la balanza deficitaria del primero y la superavitaria del segundo.

Se aprecia que la tendencia comercial para ambos países es positiva; sin embargo, el comercio de México está creciendo a un ritmo mucho más elevado que el de Nueva Zelanda. También se puede observar que en México las exportaciones mues- 
El papel de los bosques en el Protocolo de Kyoto: el caso del manejo forestal de Nueva Zelanda y México

Cuadro 4

Comercio internacional de productos forestales de Nueva Zelanda y México: 1990-2000 (millones de dólares)

\begin{tabular}{|c|c|c|c|c|c|c|c|c|}
\hline \multicolumn{5}{|c|}{ Nueva Zelanda } & \multicolumn{4}{|c|}{ México } \\
\hline $\begin{array}{l}\text { Años/crec. } \\
\text { Prom. } \\
\text { Anual (\%) }\end{array}$ & $x$ & $M$ & Saldo & $(X+M)$ & $x$ & $M$ & Saldo & $(X+M)$ \\
\hline 1990 & 5,679 & 1,487 & 4,192 & 7,166 & 903 & 5,166 & $-4,263$ & 6,069 \\
\hline 1991 & 6,782 & 1,332 & 5,450 & 8,113 & 844 & 5,637 & $-4,793$ & 6,481 \\
\hline 1992 & 7,339 & 2,231 & 5,107 & 9,570 & 1,354 & 8,301 & $-6,946$ & 9,655 \\
\hline 1993 & 10,012 & 1,891 & 8,121 & 11,902 & 766 & 8,263 & $-7,497$ & 9,029 \\
\hline 1994 & 10,689 & 1,704 & 8,985 & 12,393 & 810 & 9,538 & $-8,728$ & 10,349 \\
\hline 1995 & 12,157 & 2,372 & 9,785 & 14,529 & 1,840 & 10,645 & $-8,805$ & 12,484 \\
\hline 1996 & 11,528 & 2,111 & 9,418 & 13,639 & 2,599 & 9,991 & $-7,392$ & 12,590 \\
\hline 1997 & 10,558 & 2,240 & 8,318 & 12,798 & 2,855 & 10,853 & $-7,998$ & 13,708 \\
\hline 1998 & 8,016 & 2,400 & 5,616 & 10,415 & 1,980 & 11,052 & $-9,072$ & 13,032 \\
\hline 1999 & 9,341 & 2,184 & 7,157 & 11,524 & 1,842 & 16,552 & $-14,709$ & 18,394 \\
\hline 2000 & 11,035 & 2,123 & 8,911 & 13,158 & 1,707 & 20,880 & $-19,173$ & 22,587 \\
\hline $\begin{array}{l}\text { Crec. } \\
\text { Prom. (\%) }\end{array}$ & 6.9 & 3.6 & 7.8 & 6.3 & 6.6 & 15.0 & 16.2 & 14.0 \\
\hline
\end{tabular}

Fuente: www.fao.org/FAOSTAT

tran un incremente mucho más lento que las importaciones, mientras que en Nueva Zelanda las importaciones son las que crecen de manera pausada. Esto indica que de alguna manera Nueva Zelanda satisface su propio consumo interno, mientras México depende del exterior.

En suma, en nuestro análisis comparativo nos encontramos dos países con manera distintas de manejar sus recursos forestales. Mientras Nueva Zelanda, durante la década de los noventa, le dio a los bosques un manejo sustentable, México hizo lo contrario, aun cuando su marco legal así lo preveía. La eficiencia de la industria productiva y el comercio internacional de Nueva Zelanda resultan sorprendentes, si consideramos que México tiene una superficie forestal siete veces mayor que la de aquel país. Es decir, en México no solamente existe un desperdicio y un abuso de los recursos forestales, sino que también se manifiesta una fuga eco- nómica considerable, ya que las variables aquí analizadas muestran que a través de toda la cadena productiva se encuentran altos grados de ineficiencia y desinterés, tanto de los propietarios de los bosques como de los empresarios y de las autoridades correspondientes. En otras palabras, en México la ley forestal no se respeta.

Evidentemente el análisis del manejo forestal es mucho más complejo que lo que muestran los datos. Existen factores determinantes para un buen o mal manejo de los bosques que se encuentran detrás de la cadena productiva; los más importantes son: el grado de corrupción de las autoridades, el desarrollo económico del país, el régimen político, el Estado de derecho y la cultura.

El análisis anterior es una muestra de dos países con opuesto manejo forestal. El caso de Nueva Zelanda se acerca mucho al ideal de manejo forestal sustentable, y el de México refleja la realidad que vive el grueso de los países del mundo. 


\section{Conclusión}

Los recursos forestales son fundamentales dentro de la estrategia de mitigación prevista en el Protocolo de Kyoto. Por medio de los mecanismos flexibles se incentiva a los países desarrollados para que inviertan en proyectos ecológicos en otros países (programas de plantaciones, reforestaciones o forestaciones, entre otros) con el fin de reducir los gases de efecto invernadero en la atmósfera, y tales medidas contarán para los países desarrollados como reducción de gases nacionales. Por su parte, en los países no desarrollados esta estrategia representa la oportunidad de atraer inversión limpia al interior de su territorio.

En esta suma de esfuerzos (por la cual el país desarrollado invierte en el menos desarrollado, y este último se compromete a darle el mejor uso) de los países particulares, se pretende que el ganador sea el planeta en general, debido a que el control del crecimiento de los gases de efecto invernadero es un bien público mundial.

Esta intención parece convincente; sin embargo, caben algunas acotaciones:

1. Los bosques tienen múltiples funciones que van más allá de ser simples sumideros de carbono, pues desempeñan una parte importante dentro del ciclo del agua, son generadores de oxigeno limpio, son el hábitat de un sinfín de especies de flora y fauna, hogar de algunas comunidades indígenas, fuente de recreación, valor económico, entre otras.

2. Por lo anterior, debe quedar claro que las plantaciones no son bosques, los monocultivos atienden la necesidades comerciales pero no son una alternativa como sumidero de carbono, pues su tala posterior desprende dióxido de carbono, ni tienen los beneficios de un bosque natural pues no permiten la generación de vida y la reactivación del ciclo del agua, pues al contrario, según los especialistas, los monocultivos a gran escala generan un impacto negativo en el ámbito social y ambiental. ${ }^{24}$

3. En caso de que la inversión tuviera la específica misión de reactivar los bosques naturales, su éxito no estaría asegurado, ya que alrededor de ellos existen múltiples intereses que impedirían su buen desarrollo. Lo anterior se afirma bajo el entendido de que si no se respeta la legislación interna (la más rica e importante de las fuentes formales dentro de la sociedad) como es el caso de México, poco se puede esperar de esta inversión.

4. El que se haya reforzado la permanencia de los bosques bajo el manejo sustentable en las leyes forestales nacionales no garantiza su funcionamiento, ya que aun cuando en el país se aplique correctamente el Estado de derecho, el concepto de desarrollo sustentable tiene algunas fallas de origen debido a que "se preocupa en producir en un modo más limpio, se propone consumir recursos renovables, subraya la prevención - si es posible- o la minimización de los impactos negativos, pero jamás se menciona la idea de producir menos"; ${ }^{25}$ es decir, no se propone modificar el crecimiento económico (industrialización y desarrollo) sino que se pretende seguir avanzado dentro de las limitaciones y exigencias impuestas en el cuidado ambiental. En este sentido, Capalbo señala "que no puede lograrse simultáneamente un óptimo desarrollo económico al mismo tiempo que un óptimo de sustentabilidad ambiental". ${ }^{26}$ Por ello, al no pretender disminuir la producción el medio ambiente es el que saldrá perjudicado. 
Lo que tal vez funcionaría sería algún tipo de sanción al país que hace un mal uso del bosque natural, pero recordemos que ni el Protocolo de Kyoto ni el Acuerdo para el Manejo Forestal Sustentable emitido en la Conferencia de Río están respaldados con fuerza jurídica obligatoria. En este sentido, la estrategia de mitigación por medio del aumento de la superficie forestal no se puede garantizar como exitosa. niv

\section{Notas}

1 Ludevid, 1998, pp. 240-247.

2 Samuelson y Nourdhaus, 2002, p. 320.

3 Gómez-Pompa y del Amo, 1994, p. 163.

4 www.fao.org/forestry

$5 \quad$ FAO, 1948, p. 1.

6 Giddens, 2000, p. 30.

7 Instituto Nacional de Ecología, 2003.

8 El Protocolo de Kyoto, en: http://www.appa. es/dch/protocolo_kyoto.htm

9 SNC, en: http://www.sncweb.ch/spanisch/reportajes/protocolo-kyoto.htm

10 UnFCCC, s/f.

11 Véase Rosenbaum, Schoene, Mekouar, 2004.

12 Focus, 2004.

13 Cepal/Pnuma, 2001, p. 297.

14 Bárcena, Ibarra y Subyaga, 2000, p. 12.

15 Field y Field, 2002, p. 37.

16 Ídem.

17 Véase fao (s/f) Los desafios del cambio climático global, en: http://www.fao.org/Regional/LAmerica/prior/recnat/clima.htm

18 FAO, 2001, p. 1.

19 FAo, 1997.

20 Véase Nueva legislación de tierras, bosques y aguas.

21 http://www.fao.org/forestry/foris/webview/forestry2/index.jsp?sitetreeId=18927\&langId=1\&ge $\mathrm{oId}=0$

22 WRM, 2000, p. 1.

23 FAO, 2003, p. 3.

24 "La fauna autóctona empieza a escasear en el área y en las cercanías de las plantaciones es casi nula. Los cambios en el ciclo hidrológico producen escasez de agua y en algunos casos, incluso, dan lugar a inundaciones superiores a lo normal, luego de intensas lluvias. Las plantas útiles desaparecen. Los cursos de agua se deterioran por causa de la sedimentación resultante de la erosión de suelos generada por las plantaciones. El manejo de las plantaciones genera contaminación química por intenso uso de agroquímicos. Estos cambios tienen fuertes implicaciones para los medios de las poblaciones locales" (http://www.wrm.org.uy/plantaciones/ material/impactoscopia.html).

25 Capalbo, 2000.

26 Ídem.

\section{Bibliografía}

Blasco, Emilio (s/f) Ante el primer decenio de la Conferencia de Río de Janeiro sobre el Medio Ambiente y Desarrollo, en: http://www.ccee.edu.uy/ensenian/ catderpu/material/medioambiente.PDF

Capalbo, Lucio (2000) "Desarrollo: del dominio material al dominio de las ilimitadas potencialidades humanas", El resignificado del desarrollo. Buenos Aires: Consejo Editorial de UNIDA.

CEPAL/PNUMA (2001) Equidad, desarrollo y ciudadanía. Bogotá: Alfaomega.

FAO (1948) "Las reservas forestales del mundo", Unasylva, vol. 2. Roma: FAO.

_ (1997) "In Depth Country Study-New Zealand". Roma: Forestry Policy and Planning Division.

Field y Field (2002) Economía ambiental. España: McGraw-Hill.

Focus (2004) Cambio climático, poblaciones rurales y recursos forestales, núm. 2, FAO.

Giddens, Anthony (2000) Un mundo desbocado. España: Taurus.

Instituto Nacional de Ecología (s/f) La Convención Marco de las Naciones Unidas sobre Cambio Climático, en: http://www.ine.gob.mx/dgicurg/ cclimatico/cmnucc.html

Ludevid, Manuel (1998) El cambio global del medio ambiente. México: Alfaomega.

Rosenbaum, Schoene, y Mekouar (2004) Climate Change and the Forest sector. Roma: FAO.

Samuelson y Nordhaus (s/f) Economía, $17^{a}$ edición. México: McGraw-Hill.

Téllez, Luis (coord.) (1993) Nueva legislación de tierras, bosques y aguas. México: Fondo de Cultura Económica.

UNFCCC (s/f) Información básica del cambio climático, en: http://unfccc.int/portal_espanol/essential_ background/items/3336.php

http://www.fao.org/forestry/foris/webview/forestry2/ index.jsp? sitetreeld $=18927$ \&langld $=1$ \&geold $=0$

www.fao.org/FAOSTAT 